\title{
Supplementary Information: Phosphate ions alter the binding of daptomycin to living bacterial cell surfaces
}

\author{
Lindsey N. Miller, Marea J. Blake, Eleanor F. Page, Hannah B. Castillo \\ and Tessa R. Calhoun* \\ Department of Chemistry, University of Tennessee, Knoxville, TN 37966, \\ United States \\ *trcalhoun@utk.edu
}

\section{Table of Contents:}

S2 Second Harmonic Generation

S3 Langmuir Adsorption Isotherms and Models

S3 Cartoon schematic of the daptomycin adsorption onto the membrane. (Figure S1)

S5 SHG isotherms of initial daptomycin adsorption onto E. faecalis cells and a comparison of different model fits. (Figure S2)

S5 Comparison of extracted equilibrium constants for Langmuir vs. modified Langmuir model fitting from the data in Figure S1. (Table S1)

S6 SHG intensity from sterile calcium chloride titration into sterile 0.1 M PBS blank solution. (Figure S3)

S7 SHG isotherms of initial daptomycin adsorption onto E. faecalis cells when using different Dap: $\mathrm{Ca}^{2+}$ ratio. (Figure $\mathrm{S} 4$ )

S8 Extended SHG isotherms of initial daptomycin adsorption studies in a $0.9 \% \mathrm{NaCl}$ environment using a constant 1:8 Dap: $\mathrm{Ca}^{2+}$ ratio with E. faecalis cells and blank controls. (Figure S5)

S9 DLS size measurements of calcium-phosphate aggregates in 0.01 M PBS. (Figure S6)

S10 SHG isotherms of initial daptomycin adsorption onto E. faecalis cells with and without $2 \%$ OxyRase. (Figure S7)

S11 References 


\section{Second Harmonic Generation}

When light with a frequency $\omega$ is incident on a population of molecules a polarization is induced that oscillates at the fundamental frequency $\omega$ but also higher orders of this frequency. Second harmonic generation arises from the second-order polarization of the sample at $2 \omega$. The electric field corresponding to this generated signal is represented as:

$$
E_{2 \omega} \propto P_{2 \omega}^{(2)}=\chi_{e f f}^{(2)} E_{\omega} E_{\omega}
$$

where $\chi_{e f f}^{(2)}$ is the effective second order susceptibility and $E_{\omega}$ is the driving laser field.

The effective second order susceptibility can further be expressed as:

$$
\chi_{e f f}^{(2)} \propto N_{s} \sum_{k, e} \frac{\mu_{g k} \mu_{k e} \mu_{e g}}{\left(\omega_{g k}-\omega-i \Gamma\right)\left(\omega_{e g}-2 \omega-i \Gamma\right)}
$$

where $N_{s}$ is the number of molecules contributing to the SHG process, $\mu_{g k}, \mu_{k e}$, and $\mu e g$ are the transition dipole moments between the electronic states of the molecules, $\omega_{g k}$ and $\omega_{e g}$ are the corresponding transition frequencies and $\Gamma$ is the transition line width. Here the resonant enhancement can be seen as $\omega_{g k}$ approaches $\omega$ and/or $\omega_{e g}$ approaches $2 \omega$.

In our experiments, SHG originates from the daptomycin molecules embedded in the cell membranes. The specific chromophore is the kynurenine residue with a weak absorption peak at $380 \mathrm{~nm}$ that tails into $400 \mathrm{~nm}$, resonant with the SHG from our $800 \mathrm{~nm}$ incident laser. The signal from molecules on the same cell add coherently while signal from different cells is incoherent ${ }^{1}$. The coherent sum two different molecules $(a$ and $b$ ) molecules on the same cell can be expressed $a^{2}$

$$
I_{2 \omega}=\left|E_{a}+E_{b}\right|^{2}=2\left|E_{a}\right|^{2}+\left|E_{b}\right|^{2}+\left|E_{a}\right|\left|E_{b}\right|\left\langle\cos \left(\phi_{a}-\phi_{b}\right\rangle\right.
$$

where $\phi_{a}$ and $\phi_{b}$ are defined as the angles of the emitted electric fields relative to the membrane axis. These angles are determined by the dipole moments and thus the relative orientation of the molecules. As the alignment of the molecules is matched relative to the membrane, the SHG signal increases.

Finally, it should be noted that effective second order susceptibility can also be expressed as

$$
\chi_{e f f}^{(2)} \propto N_{s}\langle\beta\rangle
$$

where the scattering of each molecule is described as a point dipole with the hyperpolarizability, $\beta .\langle\beta\rangle$ is then the average orientation of the total population ${ }^{3,4}$.

\section{Langmuir Adsorption Isotherms and Models}

Langmuir adsorption isotherms can be measured using SHG spectroscopy without the addition of exogenous labels to determine and compare daptomycin's binding affinities for cell membranes while in various environmental conditions, essentially revealing daptomycin's unaltered initial interactions with living bacterial systems. Before now, past daptomycin isotherm studies required the use of model membrane systems or fluorescent $\operatorname{tags}^{5-8}$. SHG 


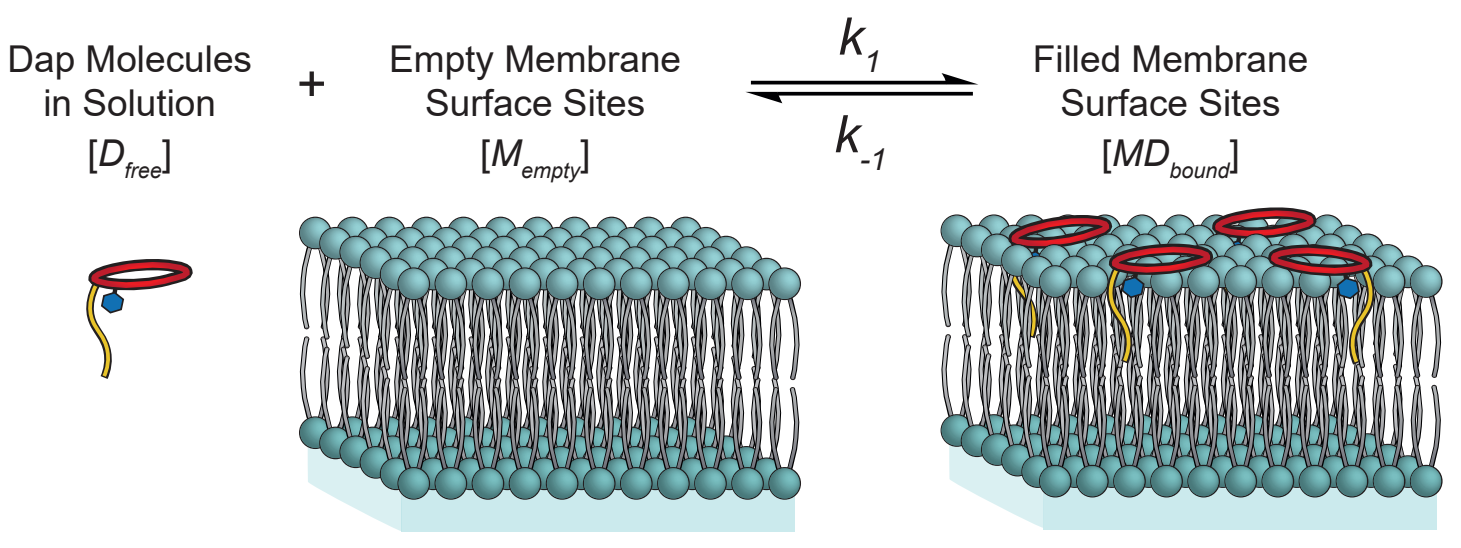

Figure S1: Cartoon schematic of the daptomycin adsorption onto the membrane. Not shown are the $\mathrm{Ca}^{2+}$ ions that are known to be necessary but whose exact role is still debated.

Langmuir adsorption isotherms have been previously employed to assess the binding affinities for different small molecule-membrane systems such as in model membranes ${ }^{9-16}$ and in living cell membranes ${ }^{17-19}$ The cartoon schematic in Fig. S1 represents the adsorption of free daptomycin molecules in solution, $D_{\text {free }}$, onto empty membrane sites, $M_{e m p t y}$, and the resulting production of daptomycin-bound membrane sites $M D_{\text {bound }}$ that generate the measured SHG signal.

In our experiments, the concentration of daptomycin is increased while monitoring the SHG response to assess the availability of empty membrane sites, $M_{\text {empty }}$. The results can be fit by the equation:

$$
\theta=\frac{\frac{[c]}{K_{d}}}{1+\frac{[c]}{K_{d}}}
$$

where $c$ is the concentration of daptomycin and $\theta$ represents the fraction of $M D_{\text {bound }}$ to $M_{\text {total }}$, such that:

$$
\theta=\frac{M D_{\text {bound }}}{M_{\text {total }}}
$$

$K_{d}$ in Eq. S5 is the dissociation constant $\left(K_{d}=\frac{k_{-1}}{k_{1}}\right)$ and represents the concentration at which half of the membrane sites are occupied ${ }^{1}$. A smaller $K_{d}$ value corresponds to a stronger association with the membrane.

While the application of the standard Langmuir model as presented in Eq. S5 is a common analysis in the field, ${ }^{19-21}$ an alternative approach is to use the modified Langmuir model: ${ }^{11,14,17,22}$

$$
\theta=\frac{\left(C+N_{\max }+\frac{55.5}{K_{a}}\right)-\sqrt{\left(C+N_{\max }+\frac{55.5}{K_{a}}\right)^{2}-4 C N_{\max }}}{2 N_{\max }}
$$

where $C$ is the bulk concentration of the adsorbate species, $N_{\max }$ is the maximum number of sites available on the surface, $K_{a}$ is the equilibrium constant for association, and 55.5 is the molarity of water. This equation is the more complete form of the Langmuir model and accounts for the potential depletion of the adsorbing species from the bulk solution. 
There are a few other details that should be pointed out when quantitatively comparing the extracted parameters from the modified Langmuir model to those resulting from standard Langmuir equation provided in the main text. Specifically, Eq. S7 is expressed in terms of the association constant, $K_{a}$, which is the inverse of the dissociation constant, $K_{d}$, in our expression of standard Langmuir in Eq. S5. In addition, the modified Langmuir expresses the surface coverage as a function of the molar fraction of the solute species resulting in a dimensionless equilibrium constant. In contrast, we use the bulk concentration for our Langmuir analysis which results in the concentration units in our reported $K_{d}$ values. For more direct comparison, the standard Langmuir model can be expressed as: ${ }^{22}$

$$
\theta=\frac{[c] K_{a}}{55.5+[c] K_{a}}
$$

Figure S2 and Table S1 show a direct comparison between these fits. As can be clearly observed, we see no significant difference when using the modified Langmuir model relative to the standard Langmuir. We have chosen to report our results using the standard Langmuir model because, by providing an adequate fit with fewer variable parameters, we avoid overfitting the data. This is especially important for this system when considering that very little is actually known about what the maximum number of available sites $\left(N_{\max }\right)$ should be making it difficult to accurately restrict or evaluate this parameter. 


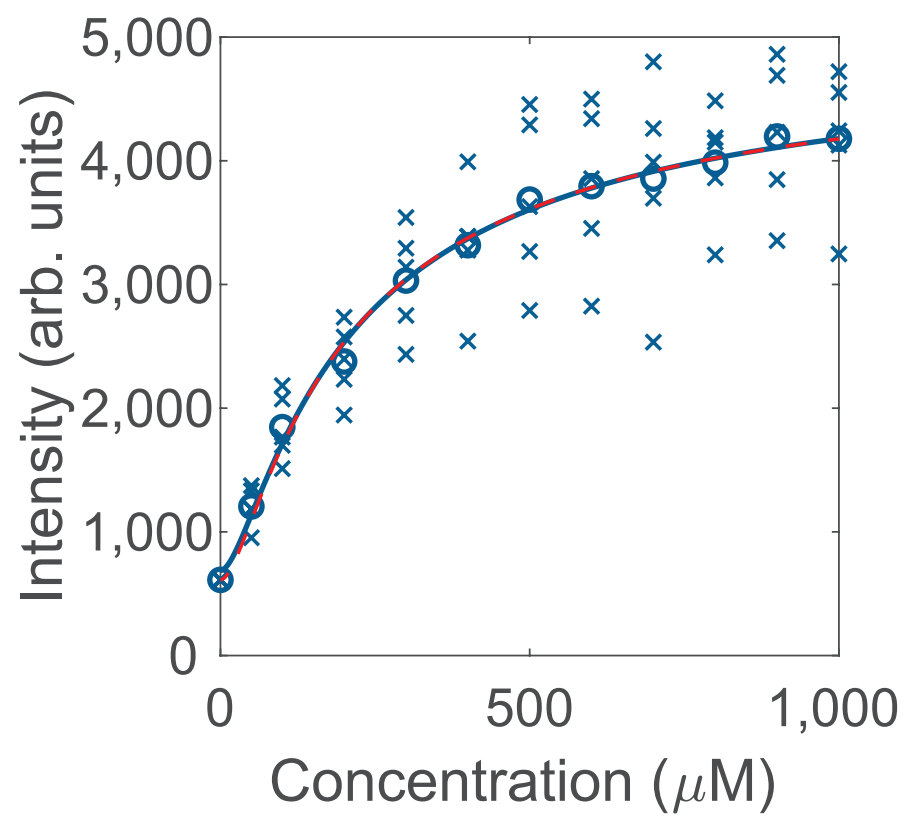

Figure S2: SHG isotherms of initial daptomycin adsorption onto E. faecalis cells and a comparison of different model fits. Trials were performed in a PBS environment using a 1:8 Dap: $\mathrm{Ca}^{2+}$ ratio. The individual trials are shown as $\times$. The averages for the curve fits are shown as $\circ$. The blue line shows the fit using the standard Langmuir model while the red line is the fit from the modified Langmuir model.

Table S1: Comparison of extracted equilibrium constants for Langmuir vs. modified Langmuir model fitting from the data in Figure S1.

\begin{tabular}{lll}
\hline Model Function & $\boldsymbol{K}_{d}(\mu \mathbf{M})$ & $\boldsymbol{K}_{a}$ \\
\hline Langmuir & $104 \pm 9$ & $0.5 \pm 0.05$ \\
Modified Langmuir & $99 \pm 12$ & $0.6 \pm 0.06$ \\
\hline
\end{tabular}




\section{SI Figures}

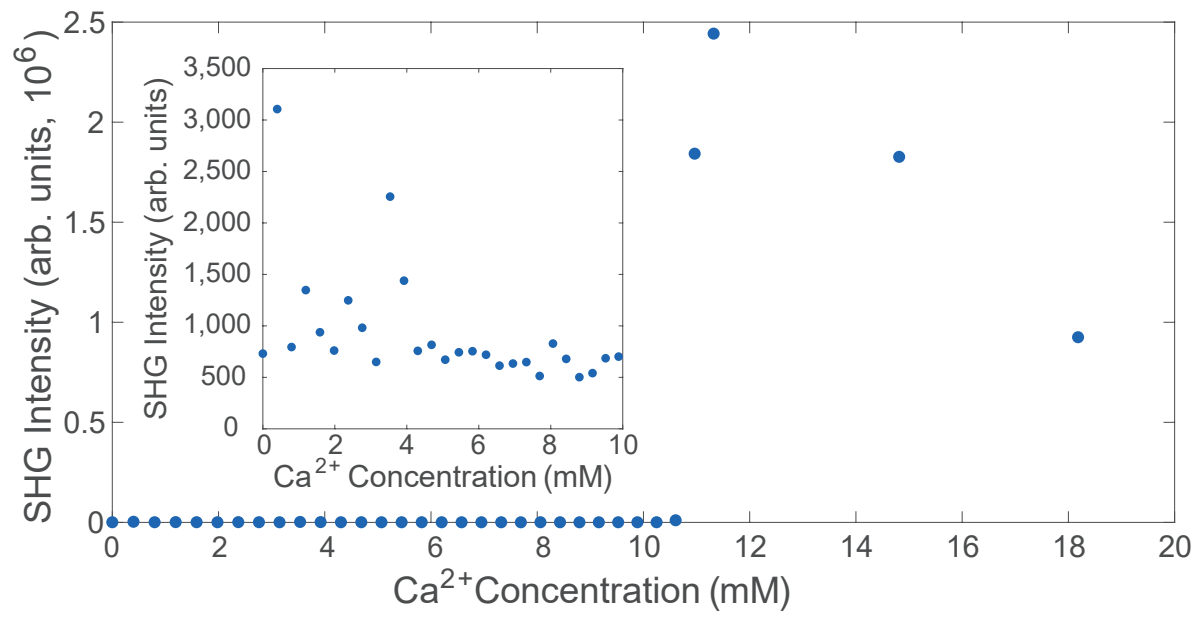

Figure S3: SHG intensity from sterile calcium chloride titration into sterile 0.1 M PBS blank solution. Inset data plot is the zoomed in region for the lower $\mathrm{Ca}^{2+}$ concentrations up to 10 $\mathrm{mM}$. Fundamental laser power was $350 \mathrm{~mW}$. Integration time was $250 \mathrm{~ms}$ and total signal collection time for each data point was $3 \mathrm{~s}$. 


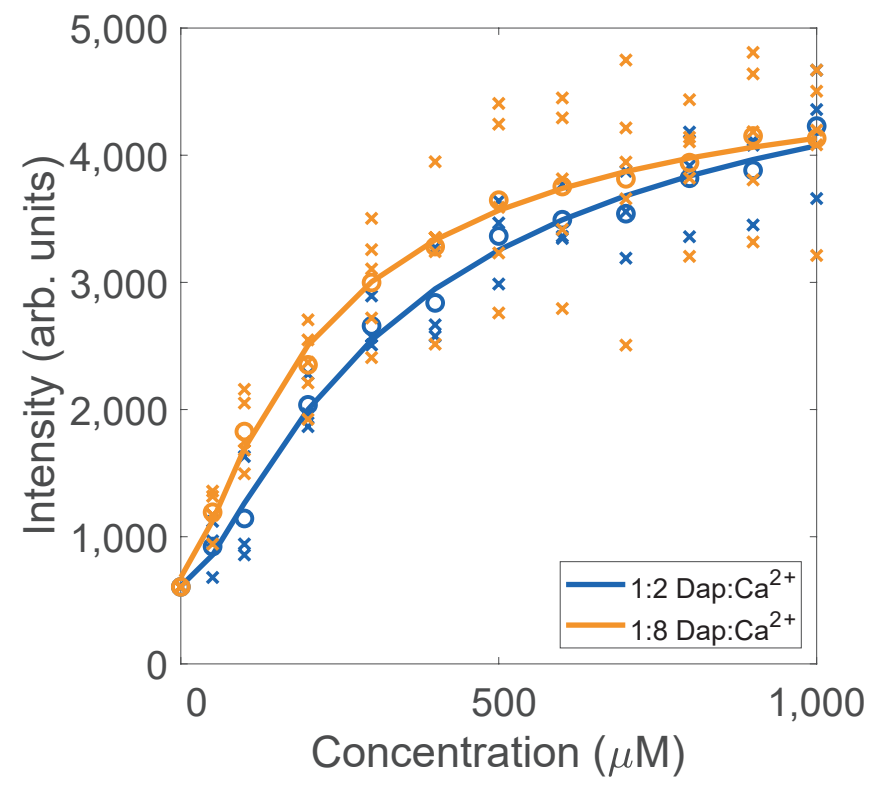

Figure S4: SHG isotherms of initial daptomycin adsorption onto E. faecalis cells when using a Dap: $\mathrm{Ca}^{2+}$ ratio of 1:2 (blue data) and 1:8 (gold data). Trials were performed in a PBS environment. The individual trials are shown as $\times$. The averages for the curve fits are shown as 0 . 


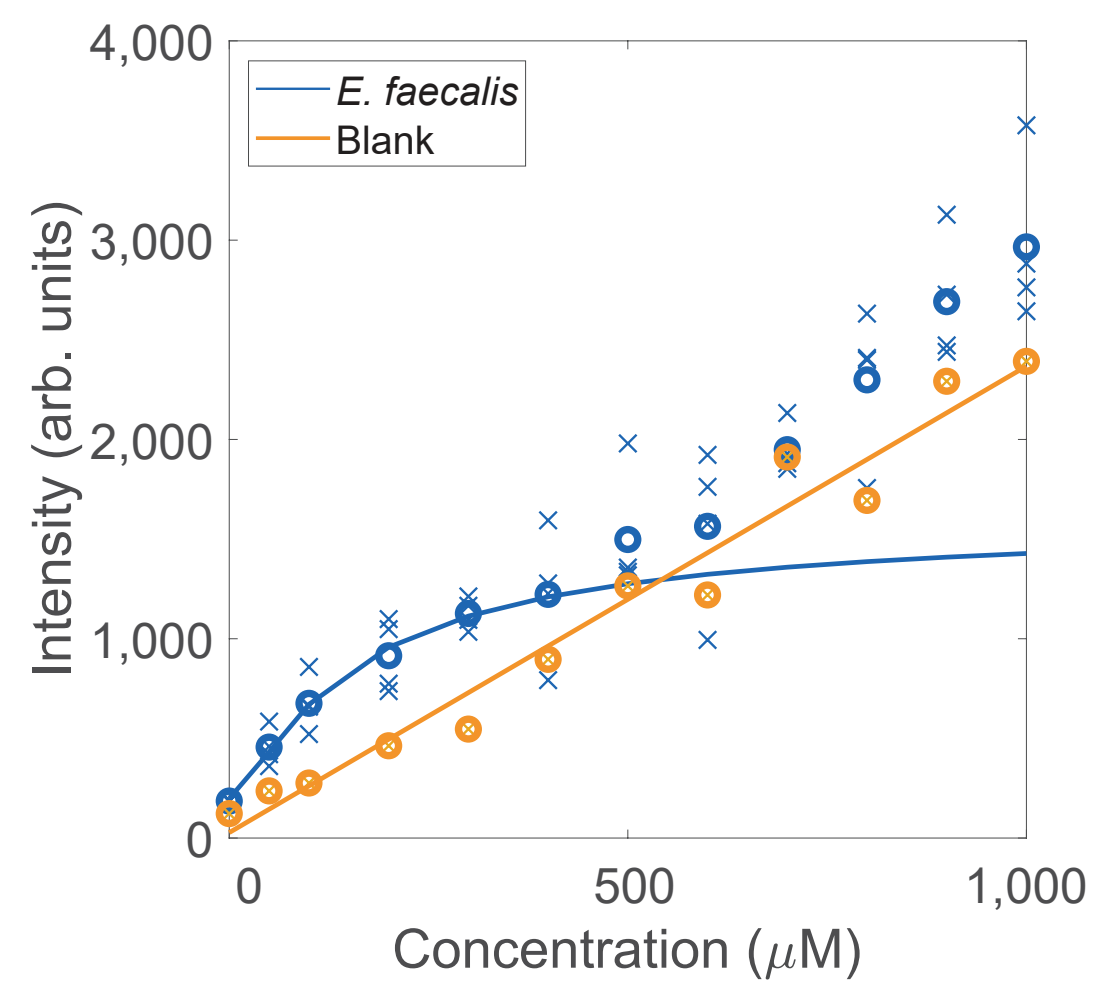

Figure S5: SHG isotherms of initial daptomycin adsorption studies in a $0.9 \% \mathrm{NaCl}$ environment using a constant 1:8 Dap: $\mathrm{Ca}^{2+}$ ratio with E. faecalis cells $(-)$ and blank controls with no cells present $(-)$. The individual trials are shown as $\times$. The averages for the curve fits are shown as $\circ$. 


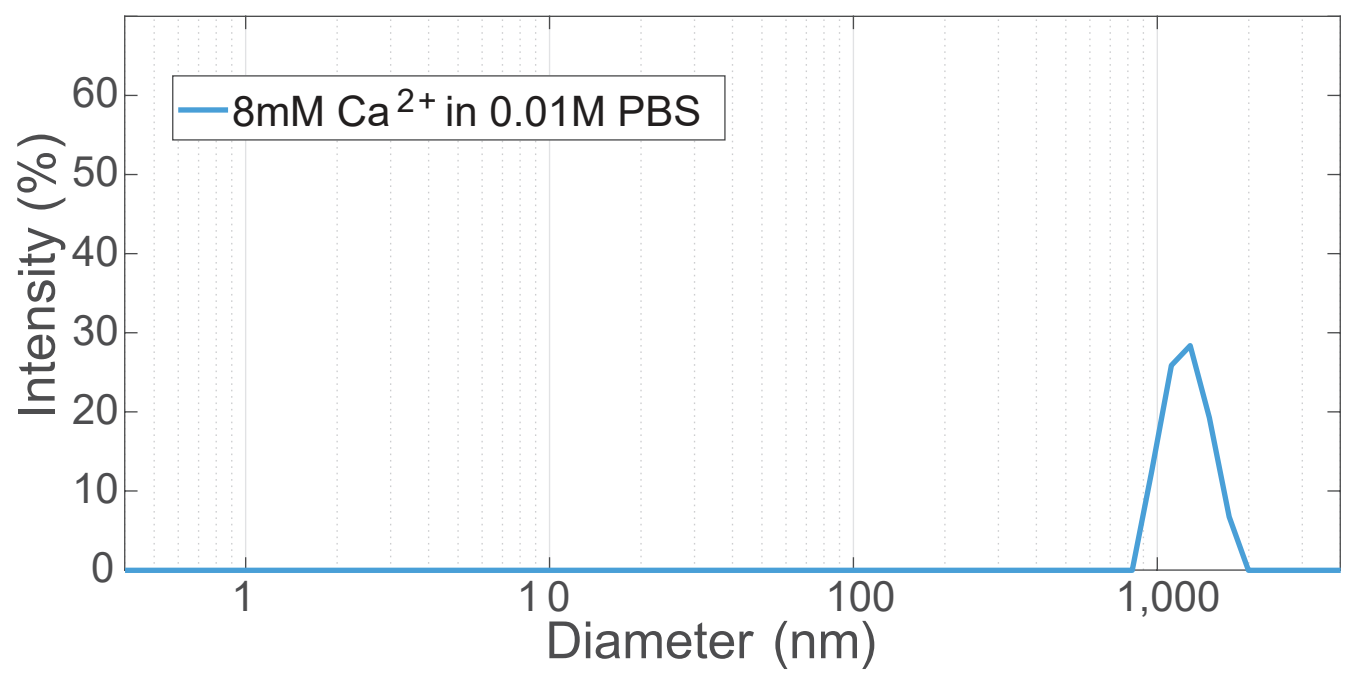

Figure S6: DLS size measurements of calcium-phosphate aggregates in $0.01 \mathrm{M}$ PBS. The calcium concentration was $8 \mathrm{mM}$. 


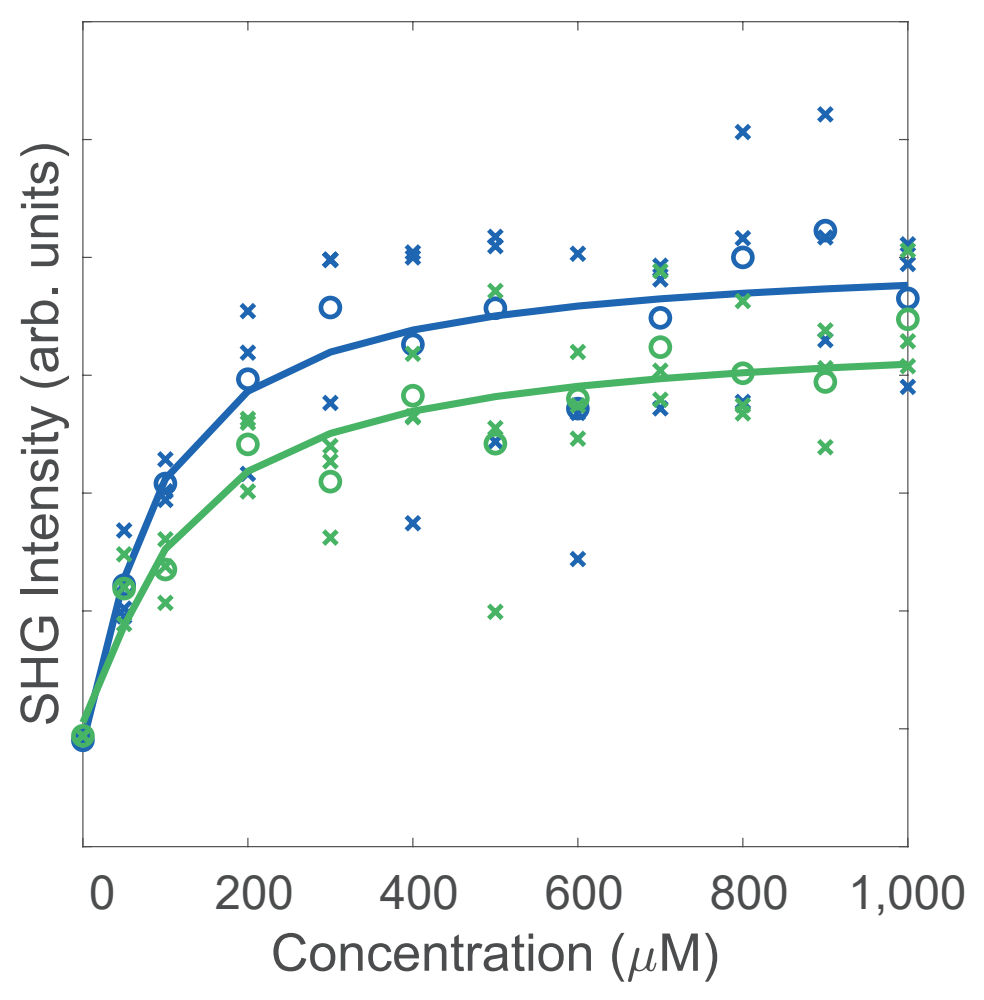

Figure S7: SHG isotherms of initial daptomycin adsorption onto E. faecalis cells with (blue data) and without (green data) 2\% OxyRase present. Trials were performed in a PBS environment using a 1:8 Dap: $\mathrm{Ca}^{2+}$ ratio. The individual trials are shown as $\times$. The averages for the curve fits are shown as o, and the lines are fits to the Langmuir model. While averaged data appears lower for the isotherm without OxyRase, as indicated by the solid lines, there is clearly overlap of the data trials for both conditions at almost all concentrations tested. This suggests that any difference caused by the presence of OxyRase is within the error of our measurement. 


\section{References}

(1) Eisenthal, K. B. Second Harmonic Spectroscopy of Aqueous Nano- and Microparticle Interfaces. Chem. Rev. 2006, 106, 1462-1477.

(2) Doughty, B.; Rao, Y.; Kazer, S. W.; Kwok, S. J. J.; Turro, N. J.; Eisenthal, K. B. Probing the Relative Orientation of Molecules Bound to DNA through Controlled Interference Using Second-Harmonic Generation. PNAS 2013, 110, 5756-5758.

(3) Roke, S.; Gonella, G. Nonlinear Light Scattering and Spectroscopy of Particles and Droplets in Liquids. Annual Review of Physical Chemistry 2012, 63, 353-378.

(4) Moreaux, L.; Sandre, O.; Mertz, J. Membrane Imaging by Second-Harmonic Generation Microscopy. J. Opt. Soc. Am. B, JOSAB 2000, 17, 1685-1694.

(5) Kinouchi, H.; Onishi, M.; Kamimori, H. Lipid Membrane-Binding Properties of Daptomycin Using Surface Plasmon Resonance. Analytical Sciences 2013, 29, 297-301.

(6) Pokorny, A.; Khatib, T. O.; Stevenson, H. A Quantitative Model of Daptomycin Binding to Lipid Bilayers. The Journal of Physical Chemistry B 2018, 122, 9137-9146.

(7) Taylor, R.; Butt, K.; Scott, B.; Zhang, T.; Muraih, J. K.; Mintzer, E.; Taylor, S.; Palmer, M. Two Successive Calcium-Dependent Transitions Mediate Membrane Binding and Oligomerization of Daptomycin and the Related Antibiotic A54145. Biochimica et Biophysica Acta (BBA) - Biomembranes 2016, 1858, 1999-2005.

(8) Lee, M.-T.; Hung, W.-C.; Hsieh, M.-H.; Chen, H.; Chang, Y.-Y.; Huang, H. W. Molecular State of the Membrane-Active Antibiotic Daptomycin. Biophysical Journal 2017, 113, 82-90.

(9) Nguyen, T. T.; Conboy, J. C. High-Throughput Screening of Drug-Lipid Membrane Interactions via Counter-Propagating Second Harmonic Generation Imaging. Analytical Chemistry 2011, 83, 5979-5988.

(10) Salafsky, J. S.; Eisenthal, K. B. Second Harmonic Spectroscopy: Detection and Orientation of Molecules at a Biomembrane Interface. Chemical Physics Letters 2000, 319, 435-439.

(11) Rao, Y.; Kwok, S. J. J.; Lombardi, J.; Turro, N. J.; Eisenthal, K. B. Label-Free Probe of HIV-1 TAT Peptide Binding to Mimetic Membranes. Proceedings of the National Academy of Sciences 2014, 111, 12684-12688.

(12) Tran, R. J.; Sly, K. L.; Conboy, J. C. Applications of Surface Second Harmonic Generation in Biological Sensing. Annual Review of Analytical Chemistry 2017, 10, 387-414.

(13) Stokes, G. Y.; Conboy, J. C. Measuring Selective Estrogen Receptor Modulator (SERM)-Membrane Interactions with Second Harmonic Generation. Journal of the American Chemical Society 2014, 136, 1409-1417. 
(14) Kumal, R. R.; Nguyenhuu, H.; Winter, J. E.; McCarley, R. L.; Haber, L. H. Impacts of Salt, Buffer, and Lipid Nature on Molecular Adsorption and Transport in Liposomes as Observed by Second Harmonic Generation. The Journal of Physical Chemistry C 2017, 121, 15851-15860.

(15) Kriech, M. A.; Conboy, J. C. Label-Free Chiral Detection of Melittin Binding to a Membrane. Journal of the American Chemical Society 2003, 125, 1148-1149.

(16) Kriech, J. C. C.; A., M. Measuring Melittin Binding to Planar Supported Lipid Bilayer by Chiral Second Harmonic Generation. Analytica Chimica Acta 2003, 496, 143-153.

(17) Zeng, J.; Eckenrode, H. M.; Dounce, S. M.; Dai, H.-L. Time-Resolved Molecular Transport across Living Cell Membranes. Biophysical Journal 2013, 104, 139-145.

(18) Zeng, J.; Eckenrode, H. M.; Dai, H.-L.; Wilhelm, M. J. Adsorption and Transport of Charged vs. Neutral Hydrophobic Molecules at the Membrane of Murine Erythroleukemia (MEL) Cells. Colloids and Surfaces B: Biointerfaces 2015, 127, 122-129.

(19) Miller, L. N.; Brewer, W. T.; Williams, J. D.; Fozo, E. M.; Calhoun, T. R. Second Harmonic Generation Spectroscopy of Membrane Probe Dynamics in Gram-Positive Bacteria. Biophysical Journal 2019, 117, 1419-1428.

(20) Nguyen, T. T.; Sly, K. L.; Conboy, J. C. Comparison of the Energetics of Avidin, Streptavidin, NeutrAvidin, and Anti-Biotin Antibody Binding to Biotinylated Lipid Bilayer Examined by Second-Harmonic Generation. Anal. Chem. 2012, 84, 201-208.

(21) Doughty, B.; Rao, Y.; Kazer, S. W.; Kwok, S. J. J.; Turro, N. J.; Eisenthal, K. B. Binding of the Anti-Cancer Drug Daunomycin to DNA Probed by Second Harmonic Generation. The Journal of Physical Chemistry B 2013, 117, 15285-15289.

(22) Wang, H.; Yan, E. C. Y.; Liu, Y.; Eisenthal, K. B. Energetics and Population of Molecules at Microscopic Liquid and Solid Surfaces. J. Phys. Chem. B 1998, 102, 4446-4450. 